\title{
Basic Classes Of Mathematical Models Used In Machine Vision Problems
}

\author{
Yeromina Nataliia ${ }^{1}$ \\ Lukashyn Oleksii ${ }^{2}$
}

${ }^{1}$ Kharkiv National University of Radio Electronics, 14 Nauky Ave, KharkivUA-61166, Ukraine, nataliia.yeromina@nure.ua

${ }^{2}$ Kharkiv National University of Radio Electronics, 14 Nauky Ave, KharkivUA-61166, Ukraine, oleksii.lukashin@nure.ua

\begin{abstract}
Today, computing platforms are a booming industry. However, a huge gap in the technology of "artificial intelligence" and its important component parts understanding scenes and images - is, in fact, a major limiting factor for further development of complex control systems. In this article we consider the basic classes of mathematical models used in the development of practical image analysis systems currently.
\end{abstract}

Keywords: machine vision, image analysis, simulation modeling, abstract modeling, mathematical model.

\section{INTRODUCTION AND PROBLEM STATEMENT}

Machine vision is a scientific field in the field of artificial intelligence and robotics, as well as related technologies for obtaining images of real-world objects, their processing and use of the obtained data to solve various kinds of applied problems without human intervention.

In various technical problems in the field of machine vision chosen certain basic ways of describing reality. Let's see in which cases it is better to use some methods, and in which others. There is no single universal solution here, but at the most general level, as two "extreme" poles, two basic fundamentally different approaches can be mentioned here: simulation (physical) modeling and abstract (brightnessgeometric) modeling[1].

Simulation in the field of machine vision involves an attempt to describe some real physical object and a real physical device for acquiring an image of this object. The advantage of this approach is the completeness and reliability of the simulation results, as well as the guaranteed quality of image analysis methods based on simulation models. The disadvantages of the simulation approach are associated with a disproportionate amount of labor at the stage of compiling the model and too much experimental data needed to build the simulation model.

Abstract modeling proceeds from the most general considerations about the nature of the analyzed objects and the way they are registered. Such an approach in the literature is sometimes called iconic [2]. The advantages of the iconic approach are that when developing image analysis methods, a minimum of a priori information about a real observation situation is required. The main drawback of abstract modeling is determined by the high probability that a method developed for a too general model will turn out to be either generally inoperative or substantially less effective in practice than methods created based on models that are specific to this task.

In practice, both approaches are rarely found in their pure form. Often, developers start from general abstract models, which are then gradually concretized and adapted to the available real data, thereby approaching simulation models. At the same time, it must be considered that the same methods and models, depending on the field of application, can pass from the class of simulation models to the class of abstract ones, and vice versa [3].

\section{PROBLEM SOLUTION AND RESULTS}

Consider the basic classes of mathematical models used in image analysis, their differences and peculiarities.

Image as a function of vector argument

Often, the initial description of an image in practice is a two-dimensional intensity function.

Many methods of image analysis, in which images are considered as two-dimensional functions, suggest that the concepts of addition and multiplication of functions, multiplication of a function by a number, scalar product of functions, norm of a function, linear space, etc. are defined. All these concepts can be transferred to the domain image analysis all known methods and results from the field of linear algebra and vector spaces [2].

It is also often assumed that function images are the required number of times continuously integrable and differentiable. This allows you to transfer to the field of image analysis all known methods and results from the field of functional analysis [4].

Representation of the image in the form of a set of points

Representation of the image in the form of a set of points makes it possible to determine the operations of union, intersection, addition, and the inclusion relation for images, thereby extending to the field of image analysis methods and results from the field of set theory [5].

\section{Image as a topological object}

An image presented as a collection of points (point pattern) can be considered as a topological object, i.e. described in terms of topological elements: connected areas, boundaries of areas, connected lines and isolated points. Accordingly, topological similarity measures and topological transformations can be defined that preserve or change in a certain way the topological properties (number and ratio of topological elements) of an image. Thus, presenting an image in the form of a list or a set of points also allows you to transfer methods and results from the field of topology to the field of image analysis. In particular, the theory of coatings, being transferred to the field of analysis of discrete binary and then grayscale images (regarded as "shadows"), led to the creation of Serre's mathematical morphology [6].

If, in addition, we are dealing with metric space, then all methods of cluster analysis are automatically applied to lists of image points [7].

Image as a geometric object

After topology and metric were introduced on the sets of points, the next step was to consider images as geometric objects.

From the field of analytic geometry, image analysis borrows the following basic elements: 
- geometric transformations of images, properties of these transformations;

- parametric description of the sets of points in the coordinate space;

- "Geometric logic" (reduction from planimetry and stereometry, rules of geometric inference);

- ways to build and find figures.

Analysis of images based on models can be considered as a kind of "generalized geometry", the differences of which from classical geometry are as follows.

- Bright-geometric aspects. Here the difference compared to the classical geometry are continuous in the transition from points, lines and surfaces to discrete lines, areas and volumes; shifting the focus of attention from simple and "right" figures to complex nonanalytic forms; the presence of the considered geometric objects of additional non-geometric characteristics (intensity, color, etc.).

- Logical-probabilistic aspects. Classical geometry never considers false, interfering, or inaccurate data. In this regard, the classical problem of substantiating the conclusions and decisions obtained also receives a broader interpretation in the analysis of images. The rationale for the decision can be strictly logical, but more often it is probabilistic or fuzzy.

- Computational aspects. The specifics of modern image analysis tasks are determined by the need to consider the specific architecture, memory size and performance of a given computer under the indicated restrictions on these parameters.

\section{Image as a set of independent features}

The image as a set of independent features represents the completion of the idea of representing the image as a set of independent informative elements. In this case, there is a transition from an arbitrary set of informative elements (geometric points, information vectors) to a more rigid structure - an ordered set of informative elements of a given size, that is, to a feature vector.

\section{Image as a structure}

Structural models of images make it possible to transfer to the field of image analysis all known methods and results from the field of structure analysis, which, however, itself was created under the significant influence of problems from the field of geometry and image analysis. In general terms, the definition of a structural model can be represented as: "a set of elements of given types that satisfy a set of conditions that describe the relationships of given types" [3].

\section{Image as a two-dimensional projection of a three-} dimensional scene.

The photogrammetric approach [8], which considers individual images and ensembles of images as two-dimensional projections of three-dimensional scenes recorded using optical systems of a specific configuration (distances and angles between cameras, passport data and distortions of camera lenses), of course, is an essential step towards simulation.
However, this approach is still not based on physical, but on geometric modeling, since it is traditionally limited by considering the geometry of the survey and does not imply an analysis of other physical factors affecting the quality of the resulting image.

The mathematical apparatus used in photogrammetry is entirely based on stereometry, projective geometry, and geometric optics.

Further, this approach can be developed by combining it with a structural approach. The task of stereo reconstruction, as well as the task of detecting and identifying objects, is thus reduced to the problem of optimal indexing of a threedimensional structural graph in an image or ensemble of stereo images [3].

Used in the modern literature on machine vision, the term high-level model approach involves solving this problem of identifying (linking) a three-dimensional structural model of an object.

\section{CONCLUSIONS}

The analysis of the subject area showed that although for each individual task of analyzing images one or the other classes of mathematical models show themselves better than the others, but for the task of localizing the binding objects of a system of mobile robots, the most universal from the point of view of flexibility and ease of use is the representation of the image in the form of a set points. flexibility and ease of use, we highlight the fundamental criteria when choosing a target mathematical model.

\section{REFERENCES}

[1] Ruban, I., Smelyakov, K., Vitalii, M., Dmitry, P., \& Bolohova, N. (2018, May). Method of neural network recognition of ground-based air objects. In 2018 IEEE 9th International Conference on Dependable Systems, Services and Technologies (DESSERT) (pp. 589-592). IEEE

[2] Yaroslavsky L.P. Digital Signal Processing in Optics and Holography: An Introduction to Digital Optics. - M.: Radio and communication, 1987.

[3] Yu.V. Wieselter, S.Yu. Zheltov, A.V. Bondarenko, M.V. Ososkov, A.V. Walrus. Image processing and analysis in machine vision tasks: Course of lectures and practical classes. - M.: Fizmatknig, 2010. - 672 pp.

[4] Lefort G. Algebra and Analysis. - M.: Science, 1973

[5] Kuratovsky K., Mostovsky A. Set theory. - M.: Mir, 1970.

[6] Serra J. Image Analysis, Mathematical Morphology. - Academic Press, 1982.

[7] R. Duda, P. Hart, Pattern Classification and Scene Analysis. - New York: John Wiley \& Sons, 1973.

[8] Lobanov A.N. Photogrammetry. - M.: Nedra, 1984. 